\title{
Physical activity and sedentary behavior among Chinese children aged 6-17 years: a cross-sectional analysis of 2010-2012 China National Nutrition and health survey
}

\author{
Chao Song, Weiyan Gong, Caicui Ding, Fan Yuan, Yan Zhang, Ganyu Feng, Zheng Chen and Ailing Liu * (D)
}

\begin{abstract}
Background: Patterns of physical activity (PA) and sedentary behavior have important implications for child health. The purpose of the study was to describe the PA and sedentary behavior among Chinese children.

Methods: The study analyzed the PA and sedentary behavior status of 38,744 Chinese children aged 6-17 years, based on the data from China National Nutrition and Health Survey (CNNHS) in 2010-2012.

Results: Chinese children spent $61.6 \mathrm{~min} / \mathrm{d}$ on school-time PA and the proportion with $60 \mathrm{~min}$ and over on schooltime PA was $52.5 \%$. The average transportation time was $38.5 \mathrm{~min} / \mathrm{d}$ and the main mode for children was walking (50.9\%), followed by taking private vehicles (19.4\%), taking public transportation (16.4\%) and cycling (13.3\%). Approximately $70 \%$ Chinese children did domestic PA and they spent $17.2 \mathrm{~min} / \mathrm{d}$ on it. Children spent $2.9 \mathrm{~h}$ per day on leisure-time sedentary behaviors and $85.8 \%$ of them engaged in sedentary behaviors longer than $2 \mathrm{~h} / \mathrm{d}$. The proportion of children participating in leisure-time PA was 35.4\%and they performed moderate-to-vigorous PA on 3.4 days per week with average 44.9 min per day. Boys were more active in school-time PA, leisure-time PA and transportation, while girls spent more time on domestic PA and homework. More older children took active transportation and spent more time on domestic PA, leisure-time PA and sedentary behaviors compared with younger children. Children in urban area were more likely to take inactive transportation and participate in leisure-time PA, spent less time on domestic PA while more time on sedentary behaviors compared with their counterpart.
\end{abstract}

Conclusions: Chinese children performed little physical activity and spent long time engaging in sedentary behaviors. Their physical activity and sedentary behaviors varied by gender, age and area.

Keywords: China, Physical activity, Sedentary behavior, Children

\section{Background}

Globally, the number of obese children and adolescents (aged five to 19 years) has risen tenfold in the past four decades [1]. In 2016, there were 50 million girls and 74 million boys with obesity in the world [2]. Owing to the well-established childhood obesity epidemic, the population prevalence of high blood pressure in the young is increasing [3]. Recently reports revealed the emerging

\footnotetext{
*Correspondence: liual@ninh.chinacdc.cn

Department of Nutrition and Health Education, National Institute for

Nutrition and Health, Chinese Center for Disease Control and Prevention, No.

27 Nanwei Road, Xicheng District, Beijing 100050, China
}

problem of type 2 diabetes in children and adolescents $[4,5]$. Accumulated evidence suggested that physical activity (PA) and sedentary behaviors were associated with physical fitness and mental health in childhood [6-9]. The World Health Organization (WHO) defined PA as any bodily movement produced by skeletal muscles that required energy expenditure, including activities undertaken while working, playing, carrying out household chores, travelling, and engaging in recreational pursuits [10]. However, a large percentage of children did not perform recommended levels of PA. Globally, $81 \%$ of the

(c) The Author(s). 2019 Open Access This article is distributed under the terms of the Creative Commons Attribution 4.0 International License (http://creativecommons.org/licenses/by/4.0/), which permits unrestricted use, distribution, and reproduction in any medium, provided you give appropriate credit to the original author(s) and the source, provide a link to the Creative Commons license, and indicate if changes were made. The Creative Commons Public Domain Dedication waiver (http://creativecommons.org/publicdomain/zero/1.0/) applies to the data made available in this article, unless otherwise stated. 
world's adolescents aged 11-17 years were insufficiently physically active in 2010 [10].

Independent of PA levels, sedentary behavior is associated with increased risk of obesity, and has a negative impact on several health outcomes [6, 11-15]. However, an ever-increasing sedentary lifestyle is becoming widespread among children [11].

China has experienced a rapid social and health transition nowadays, which made a significant impact on health and lifestyle among Chinese children, and some health related problems emerged. In Chinese schoolaged children, only 3 in 10 children achieved an "excellent" or "good" fitness standard in 2016 [16]. Meanwhile, the overweight and obesity prevalence of Chinese children aged 6-17 years were 9.6 and $6.4 \%$ respectively according to the results of China National Nutrition and Health Survey(CNNHS), which presented an increase compared with the result of China National Nutrition Survey in 2002 [17, 18]. The trend also has been reported in other Chinese studies [19, 20]. Additionally, some non-communicable disease arose among Chinese children, for instance the prevalence of hypertension was $12.4 \%$ in Chinese children aged $6-17$ years [21]. The pattern of PA and sedentary behavior was one of most fundamental risk factors. The current study was based on the data from CNNHS in 2010-2012, the largest and most comprehensive study of nutrition and health outcomes ever conducted in China. The purpose of the study was to describe the status of PA and sedentary behavior of Chinese children, investigate how PA and sedentary behavior varied by gender, age and area, then to provide basic information to aid in developing PA intervention strategies.

\section{Methods}

\section{Sampling method and implementation}

The CNNHS was a nationally representative crosssectional study conducted by the National Institute for Nutrition and Health, Chinese Center for Disease Control and Prevention (NINH, China CDC) to assess the health and nutrition status of Chinese residents. The 2010-2012 survey covered all 31 provinces, autonomous regions, and municipalities directly under the central government throughout China (except for Taiwan, Hong Kong, and Macao). The estimated sample size was 36000 children aged $6-17$ years and a multi-stage stratified cluster random sampling method was used in CNNHS. A total of 150 sites were selected, among which 34 were big cities, 41 were medium and small cities, 45 were ordinary rural areas, and 30 were poor rural areas. The four areas were classified according to the economy and social development. Big cities were the central urban areas of big cities including municipalities, 6 designated cities and capital cities (population more than one million). Medium and small cities were downtown areas that excluded the big cities. Poor rural areas were the key poor counties identified by China. Ordinary rural areas were all the rest counties outside the poor rural areas. In each site, six villages or communities were randomly selected, and in each village or community, 75 households were randomly selected.

The children aged 6-17 years in each family were involved in this survey. If the number of children in each age group was less than 20 (the number of boys and girls was equal), some children would be selected from nearby primary schools to meet the minimum sample size. Finally a total of 38,744 children aged $6-17$ years was involved and all of them completed the survey. The protocol of the 2010-2012 CNNHS was approved by the Ethical Committee of NINH, China CDC (2013-018). Signed consent forms were obtained from the children's parents or legal guardians before data collection.

\section{Physical activity and sedentary behaviors assessment}

An interview-administered questionnaire which conducted at child's home or school by trained investigators was used to collect the PA and sedentary behaviors in the past semester. For the children younger than 10 years old, questionnaires were completed with the assistance of their parents or their main caregivers. The study assessed four PA domains: school-time PA, transportation PA, leisure-time PA and domestic PA. School-time PA included physical education class, exercise during class breaks, etc. Transportation PA included walking, cycling, taking public transport (bus, subway and school bus), taking private vehicles (cars, taxi, motorcycle, electric vehicles or bicycles). Leisure-time PA included planned, structured and repetitive moderate-to-vigorous PA (MVPA) in the leisure time out of school, the purpose of which was to maintain and improve fitness and health. The total time of MVPA (exceeding $10 \mathrm{~min}$ each time) and vigorous PA (VPA, exceeding $20 \mathrm{~min}$ each time) was collected separately. Domestic PA was defined as any housework that involves physical activity, such as cleaning the house, cooking, doing laundry, and so on.

Leisure-time sedentary behaviors included watching television, using computers, playing video games, reading and doing homework in leisure time. The total time on all sedentary behaviors was collected.

\section{Statistical analysis}

The participants were divided into sub-groups according to their gender, age group and areas. Age and gender standardization were performed based on the population of China in 2009 published by National Statistics Bureau. The subgroup differences in school-time PA, transportation PA, domestic PA, leisure-time sedentary behaviors were assessed using the survey-weighted regression 
analysis. The subgroup difference in the proportions and their 95\% confidence Intervals (95\% CIs) were compared using the Rao-Scott Chi-square test. For leisure-time PA (MVPA and VPA), $t$ tests were used to compare means between different gender and age groups. Group comparisons among different areas were performed using variance analysis. The significance level was set at $p<$ 0.05 using two-sided tests.

The statistical software package SAS version 9.4 (SAS Institute Inc., Cary, NC, USA) was used for data analysis.

\section{Results}

\section{Characteristics of participants}

Table 1 presents the demographic characteristics of the participants studied. The sample size was 38744 (boys: 19631, girls: 19113) with the mean age being $11.8 \pm 3.3$ years.

\section{School-time activities}

Chinese children spent 5.2 days in school per week. Older children spent more time in school (5.3 day vs. 5.0 day, $P<0.0001)$ compared to younger ones. The average school-time PA was $61.6 \mathrm{~min}$ per school day with higher in boys than in girls $(62.3 \mathrm{~min}$ vs. $60.8 \mathrm{~min}$, $P=0.0093$ ). The proportion of children who spent more than $60 \mathrm{~min}$ on school-time PA was $52.5 \%$, and there was no significant difference between different gender, age groups and areas (Table 2).

\section{Transportation PA}

The average transportation time of Chinese children was $38.5 \mathrm{~min} / \mathrm{d}$, which was higher in older children $(P=$ $0.0494)$, and children from different areas spent different time on transportation $(P=0.0007)$ (Table 2).

Children commuted to school mainly by walking (50.9\%), followed by taking private vehicles (19.4\%), taking public transit (16.4\%) and cycling (13.3\%). The

Table 1 Basic information of participants

\begin{tabular}{ll}
\hline & $\mathrm{N}(\%)$ \\
\hline Total & 38744 \\
Bonder & \\
Girls & $19631(50.7)$ \\
Age(years) & $19113(49.3)$ \\
$6-12$ & \\
$13-17$ & $23456(60.5)$ \\
Areas & $15288(39.5)$ \\
Big Cities & \\
Medium and Small Cities & $8646(22.3)$ \\
Ordinary rural areas & $10825(27.9)$ \\
Poor rural areas & $12130(31.3)$ \\
\hline
\end{tabular}

transportation mode varied by gender, age and area as shown in Table 2.

\section{Domestic PA}

The proportion of Chinese children doing domestic PA was $69.6 \%$, which was higher in girls $(73.6 \%$ vs. $66.1 \%, P<0.0001)$, older children $(78.4 \%$ vs. $62.5 \%$, $P<0.0001)$. The average time on domestic PA was $17.2 \mathrm{~min} / \mathrm{d}$, which was higher in girls (18.9 vs. 15.7 $\mathrm{min} / \mathrm{d}, P<0.0001)$, older $(21.6$ vs. $13.6 \mathrm{~min} / \mathrm{d}, P<$ $0.0001)$ and poor rural area $(P=0.0274)$ (Table 2$)$.

\section{Leisure-time sedentary behavior}

The average time children spent on leisure-time sedentary behavior was $2.9 \mathrm{~h} / \mathrm{d}$ and the proportion of children engaging in leisure-time sedentary behavior longer than $2 \mathrm{~h} / \mathrm{d}$ was $85.8 \%$ (Table 2). The older children and the children in big cities spent more time on leisure-time sedentary behavior compared with their counterparts, while no significant difference was found between boys and girls (Table 2). Children spent nearly $1.5 \mathrm{~h} / \mathrm{d}$ on their homework which accounted for about a half of the sedentary time. Girls, older children and children in big cities spent more time on homework compared with their counterparts (Table 2).

\section{Leisure-time PA}

Table 3 shows that $35.4 \%$ of Chinese children participated in leisure-time PA. The proportion of leisure-time PA participation was higher in boys and older age group $(P<$ 0.0001 ). The highest proportion of leisure-time PA was found in children from big cities, and the lowest proportion in children from poor rural areas $(P<0.0001)$.

The children performed MVPA on 3.4 days per week with average $44.9 \mathrm{~min}$ per day, and performed VPA on 2.3 days per week. The leisure-time PA varied by gender, age groups and areas $(P<0.0001)$, except for the amount of day on MVPA between different age groups $(P=0.7778)$.

\section{Discussion}

This study clearly demonstrated different domains of PA of Chinese children. As regards of school-time PA, WHO recommended children and youth aged 5-17 should accumulate at least $60 \mathrm{~min}$ of MVPA daily [22]. This study showed that only $52.5 \%$ of children spent at least an hour on physical activity in school. This results suggested approximately half children did not meet the recommendation of $\mathrm{WHO}$, and boys spent more time doing School-time PA in comparison with girls, so Chinese children, especially girls, should be encouraged to participate PA in their leisure time.

Our data showed that Chinese children spent $38.5 \mathrm{~min}$ on their commuting, which was similar to the result of 


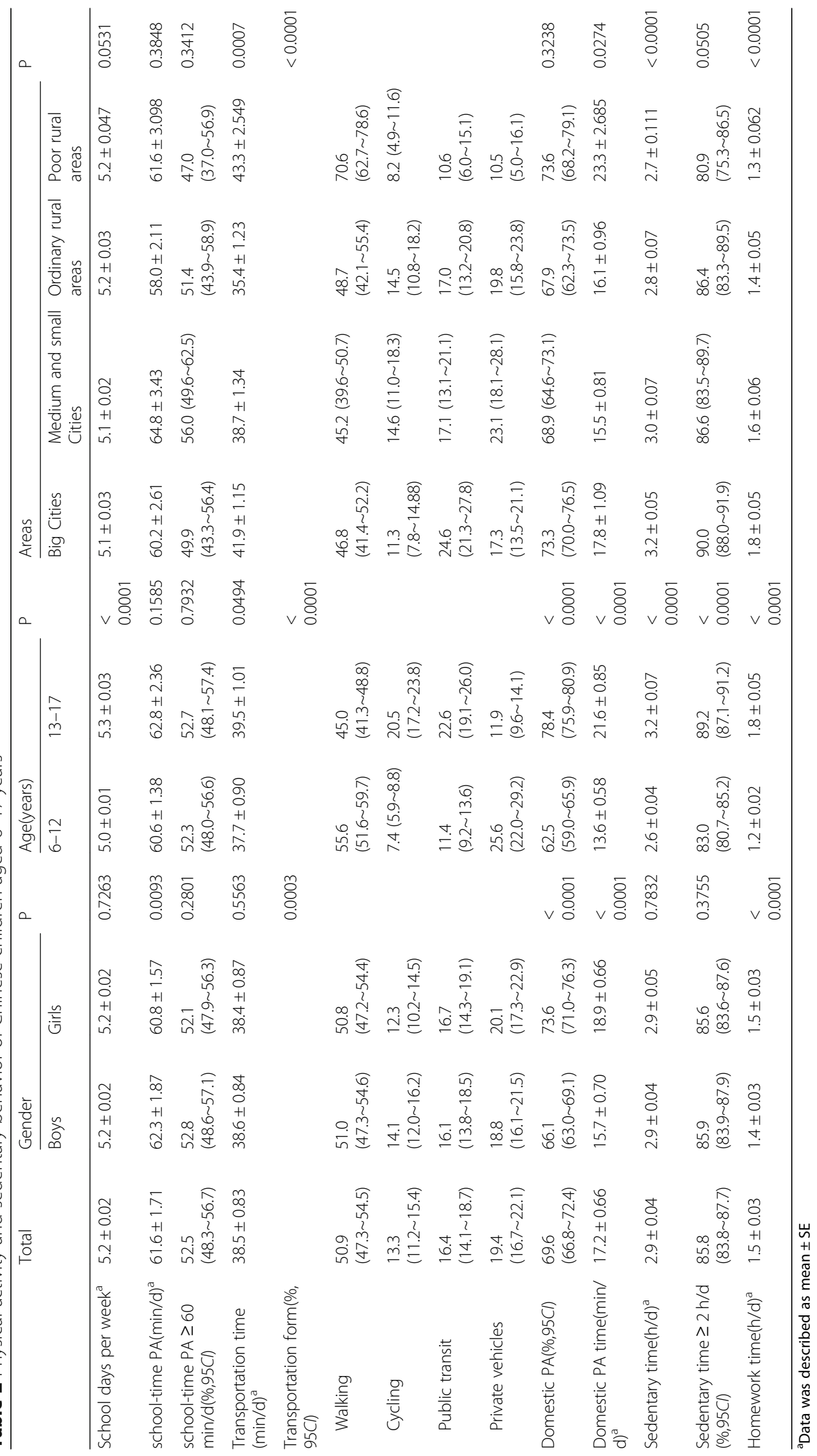




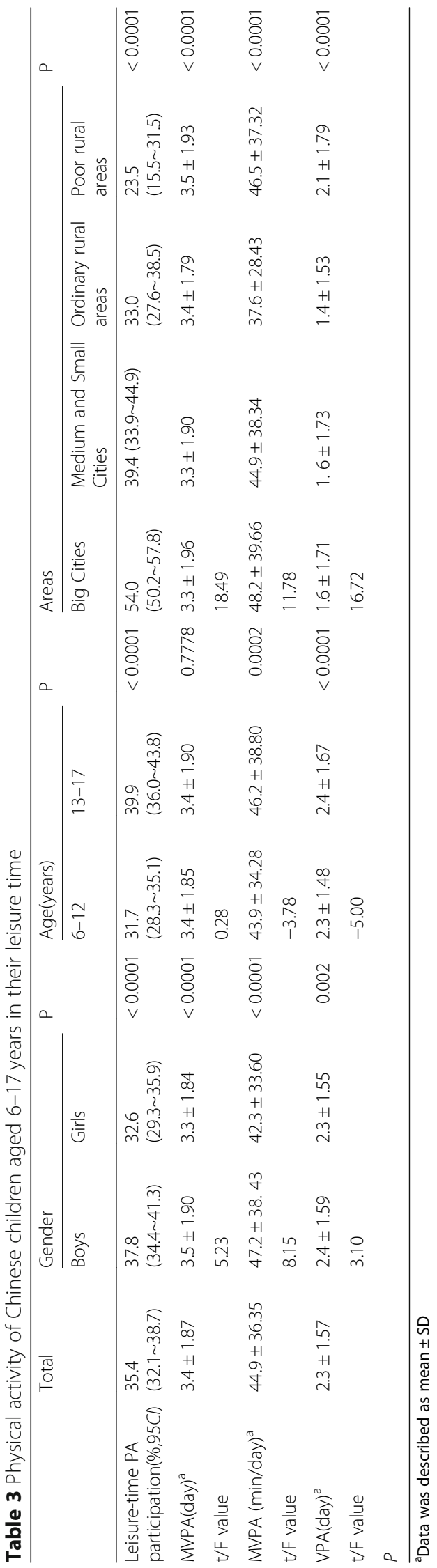


national nutritional survey in 2002 [18]. The results also showed $64.2 \%$ children walked or cycled to school, which was higher than US children and lower than that in Swiss $[23,24]$. With urbanization and economic development, a declining trend of active transport (walking and cycling) was observed internationally [23-25]. This study also showed that in comparison with children in rural areas, fewer children in urban areas went to school by active transport, while more of them went to school by motorized vehicles. Similar trends have been found in United States, New Zealand and African children [2628]. Commuting is one type of PA. It has been widely acknowledged that walking and cycling to school can contribute to accumulate PA [29-32], so these active means of school transport should be encouraged.

According to the current study, only $35.4 \%$ of Chinese children performed leisure-time PA. The data indicated that Chinese children were physically inactive in their leisure time, and the similar problem existed in many other countries: as described by Hallal e tal 2012, and has been found among students aged 13-15 in the Association of Southeast Asian Nations (ASEAN) member states, in Portuguese healthy children and adolescents aged 10-18 years, and in Scottish adolescents [33-36]. Boys participated in more PA, and which was similar in several previous studies. For example, a study conducted on a nationally representative sample of US students in grades 6-10 showed that boys reported significantly more PA than girls. A cross-sectional study including 1568 UK children aged 9-10 years showed that girls participated less in VPA and had more sedentary time than boys. Another study conducted in Tehran high schools showed that boys had significantly higher daily energy consumption than girls [37-39].

For children and adolescents, doing domestic PA can foster their problem-solving abilities and help them develop self-confidence and a sense of responsibility, so children and adolescents should undertake some housework. In the current study, approximately $70 \%$ Chinese children did domestic PA, which was higher than that of the survey in 2002 [18]. Data showed Chinese children aged 6-12 years spent $13.6 \mathrm{~min} / \mathrm{d}$ on domestic PA, which is a little higher than that in 2002 [18]. Chinese children aged $13-17$ years spent $21.6 \mathrm{~min} / \mathrm{d}$, which is lower than that in 2002 and lower than American children aged $15-17$ years $(0.78 \mathrm{~h})[18,40]$.

This study showed that Chinese children spent $2.9 \mathrm{~h}$ on leisure sedentary behaviors. Actually, in children's daily lives, there was quite a lot of time spent on sitting in classes, which was basically sedentary. If these periods were added, sedentary time would be longer. There was no significant difference between boys and girls, which was similar to the results in 2002 [18], Canadian children [41], and UK children aged 9-11 years [8]. Children in higher age groups spent more time on their sedentary behaviors and homework than the lower age groups, and this difference was also found in a New Zealand-based study and Pate's study [42, 43], which was likely due to the greater learning pressure on the senior children to learn and to achieve high grades. Children in urban areas had more leisure sedentary time than that in rural areas. In contrast, children in urban areas had a greater chance to access electronic equipment such as television, computers, smart phones and others. Similarly, a survey in Brazil found that the children in urban areas spent more time on television, video games and computers [44]. The Sport, Physical Activity, and Eating Behavior: Environmental Determinants in Young People study in the UK showed that children from higher socio-economic status families exhibited more afterschool and weekend sedentary time [45]. In addition, similar results, that affluence was a significant contributing factor to sedentary behavior, were found in Ghanaian adolescents [46]. Moreover, children in urban areas spent more time on homework than that in rural areas. This may be because urban parents paid more attention to the children's education, and their children had more homework to do. Therefore, supportive family environments need to be established to reduce sedentary time.

The data gave a general description of PA and sedentary behavior of Chinese children aged 6-17 years, and provided a basis and a reference for further intervention strategies. However, the data were collected through selfor proxy-reports, so our study suffered from reporting bias, specifically a combination of social desirability bias and the cognitive challenge associated with estimating physical activity for adults and children [47]. However, before the survey, a pilot study was performed to ensure the survey was scientific and feasible.

In China, the government have made some regulations and taken some actions. For example, School Sanitary Regulation issued by Ministry of Education and National Health Commission in 1990 and Sun Sports Programme launched in 2007. The current study indicated the level of PA among children and adolescents was still far from optimistic, especially leisure-time PA, as well as higher time of sedentary behaviors. The reason might due to the current policy mainly aimed at school-time PA, rather than the each domain of PA and sedentary behaviors. The transportation PA, leisure-time PA and domestic PA are also important components of PA and good to the development and health of children, and on the contrast, sedentary behavior is an independent risk factor. So the attention should be also paid outside the school, and the supportive environments such as the community and the family should be established. 


\section{Conclusions}

Our data suggested that only one in three children participated leisure-time PA, two in three children went to school by active transport, and about $70 \%$ children did domestic PA. Chinese children spent long time on sedentary behavior. Boys were more active to participate in PA than girls, while girls spent more time on domestic PA and homework. Older children and children in rural area were more likely to take active transportation compared with their counterparts, and older children and children in urban areas spent more time on sedentary behaviors. So a supportive environment should be established at different levels such as government, community, schools and families, to encourage children to promote their physical activity levels and reduce their sedentary behavior, especially for girls, older children and children in urban area.

\section{Abbreviations \\ 95\% Cls: Confidence Intervals; ASEAN: The Association of Southeast Asian Nations; CNNHS: China National Nutrition and Health Survey; MVPA: Moderate-to-vigorous PA; NINH, China CDC: National Institute for Nutrition and Health, Chinese Center for Disease Control and Prevention; PA: Physical activity; VPA: Vigorous PA; WHO: World Health Organization}

\section{Acknowledgments}

We thank all team members and participants for the China National Nutrition and Health Survey (CNNHS 2010-2012).

\section{Authors' contributions}

The authors' contributions were as follows: CS participated in the data clean, data check and analysis, wrote the manuscript. AL designed the study and revised the manuscript. WG, CD, FY, YZ, GF and ZC collected and supervised the data. All authors read and approved the final manuscript.

\section{Funding}

The 2010-2012 CNNHS was supported by Medical Reform Major Program from the National Health and Family Planning Commission of the People's Republic of China (No.20120212), and the funding body participated in the design of the study.

\section{Availability of data and materials}

The datasets generated and/or analyzed during the current study are not publicly available because some results are still being analyzed but are available from the corresponding author on reasonable request.

\section{Ethics approval and consent to participate}

The protocol of the 2010-2012 CNNHS was approved by the Ethical Committee of NINH, China CDC (2013-018). Signed consent forms were obtained from the children's parents or legal guardians before data collection.

\section{Consent for publication}

Not applicable.

\section{Competing interests}

The authors declare that they have no competing interests.

Received: 6 November 2018 Accepted: 28 June 2019

Published online: 11 July 2019

\section{References}

1. World Health Organization.Tenfold increase in childhood and adolescent obesity in four decades: new study by Imperial College London and WHO. http://www.who.int/en/news-room/detail/11-10-2017-tenfold-increase-in- childhood-and-adolescent-obesity-in-four-decades-new-study-by-imperialcollege-london-and-who.

2. Collaboration NRF. Worldwide trends in body-mass index, underweight, overweight, and obesity from 1975 to 2016: a pooled analysis of 2416 population-based measurement studies in 128.9 million children, adolescents, and adults. Lancet. 2017;390:2627-42.

3. Falkner B. Hypertension in children and adolescents: epidemiology and natural history. Pediatr Nephrol. 2010;25(7):1219-24.

4. Pinhas-Hamiel O, Zeitler P. The global spread of type 2 diabetes mellitus in children and adolescents. J Pediatr. 2005;146(5):693-700.

5. Dabelea D, Mayer-Davis EJ, Saydah S, Imperatore G, Linder B, Divers J, Bell R, Badaru A, Talton JW, Crume T, et al. Prevalence of type 1 and type 2 diabetes among children and adolescents from 2001 to 2009. Jama. 2014; 311(17):1778-86.

6. Biddle SJ, Asare M. Physical activity and mental health in children and adolescents: a review of reviews. Br J Sports Med. 2011;45(11):886-95.

7. lannotti RJ, Kogan MD, Janssen I, Boyce WF. Patterns of adolescent physical activity, screen-based media use, and positive and negative health indicators in the U.S. and Canada. J Adolesc Health. 2009:44(5):493-9.

8. Wilkie HJ, Standage M, Gillison FB, Cumming SP, Katzmarzyk PT. Multiple lifestyle behaviours and overweight and obesity among children aged 9-11 years: results from the UK site of the international study of childhood Obesity, Lifestyle and the environment. BMJ Open. 2016:6:e010677.

9. Saunders TJ, Gray CE, Poitras VJ, Chaput JP, Janssen I, Katzmarzyk PT, Olds T, Connor Gorber S, Kho ME, Sampson M, et al. Combinations of physical activity, sedentary behaviour and sleep: relationships with health indicators in school-aged children and youth. Appl Physiol Nutr Metab. 2016;41(6 Suppl 3):S283-93.

10. World Health Organization. Physical activity. http://www.who.int/ mediacentre/factsheets/fs385/en/.

11. Tremblay MS, LeBlanc AG, Kho ME, Saunders TJ, Larouche R, Colley RC, Goldfield G, Connor Gorber S. Systematic review of sedentary behaviour and health indicators in school-aged children and youth. Int J Behav Nutr Phys Act. 2011;8:98.

12. Shephard RJ. Sitting time and mortality from all causes, cardiovascular disease, and cancer. Yearb Sports Med. 2010;2010:124-5.

13. de Rezende LF, Rodrigues Lopes M, Rey-Lopez JP, Matsudo VK, Luiz Odo C. Sedentary behavior and health outcomes: an overview of systematic reviews. PLoS One. 2014;9(8):e105620.

14. Carson V, Hunter S, Kuzik N, Gray CE, Poitras VJ, Chaput JP, Saunders TJ, Katzmarzyk PT, Okely AD, Connor Gorber S, et al. Systematic review of sedentary behaviour and health indicators in school-aged children and youth: an update. Appl Physiol Nutr Metab. 2016;41(6 Suppl 3):S240-65.

15. Chinapaw MJ, Proper Kl, Brug J, van Mechelen W, Singh AS. Relationship between young peoples' sedentary behaviour and biomedical health indicators: a systematic review of prospective studies. Obes Rev. 2011;12(7):e621-32.

16. Zhu Z, Yang Y, Kong Z, Zhang Y, Zhuang J. Prevalence of physical fitness in Chinese school-aged children: findings from the 2016 physical activity and fitness in China-the youth study. J Sport Health Sci. 2017:6(4):395-403.

17. PRC. BoDPaCNHaFPCot: Report on Chinese Residents' Chronic Diseases and Nutrition (2015). Beijing; 2016.

18. Ma g, Kong I. Behavior and Lifestyle--The 9th Survey Report on Chinese Residents's Nutrition and Health in 2002. People's Medical Publishing House; 2002

19. Ma J, Wu S. Trend analysis of the Prevenlence of obesity and overweight among school-age children and adolescents in China. Chin J Sch Health. 2009;30(3):195-197,200.

20. Dong Y, Ma J, Song Y, Ma Y, Dong B, Zou Z, Prochaska JJ. Secular trends in blood pressure and overweight and obesity in Chinese boys and girls aged 7 to 17 years from 1995 to 2014. Hypertension. 2018;72(2):298-305.

21. Yu D, Xu X, Gao X, Fang H, Ju L, Guo H, Guo Q, Yu W, Jia F, Zhao L, et al. Status of blood pressure and prevalence of hypertension among 6-17 years old children and adolescents in 2010-2012 in China. Wei Sheng Yan Jiu. 2018:47(1):1-6.

22. World Health Organization. Global Recommendations on Physical Activity for Health. http://www.who.int/dietphysicalactivity/publications/physicalactivity-recommendations-5-17years.pdf?ua=1.

23. Grize L, Bringolf-Isler B, Martin E, Braun-Fahrlander C. Trend in active transportation to school among Swiss school children and its associated factors: three cross-sectional surveys 1994, 2000 and 2005. Int J Behav Nutr Phys Act. 2010;7:28. 
24. McDonald NC. Active transportation to school: trends among U.S. schoolchildren, 1969-2001. Am J Prev Med. 2007;32(6):509-16.

25. Buliung RN, Mitra R, Faulkner G. Active school transportation in the greate Toronto area, Canada: an exploration of trends in space and time (19862006). Prev Med. 2009;48(6):507-12.

26. Larouche R, Oyeyemi AL, Prista A, Vincent Onywera KK, Akinroye TMS. A systematic review of active transportation research in Africa and the psychometric properties of measurement tools for children and youth. Int J Behav Nutr Phys Act. 2014;11:129.

27. Babey SH, Hastert TA, Huang W, Brown ER. Sociodemographic, family, and environmental factors associated with active commuting to school among US adolescents. J Public Health Policy. 2009;30(S1):S203-20.

28. Oliver M, Badland H, Mavoa S, Witten K, Kearns R, Ellaway A, Hinckson E, Mackay L, Schluter PJ. Environmental and socio-demographic associates of children's active transport to school:a cross-sectional investigation from the URBAN study. Int J Behav Nutr Phys Act. 2014;11:70.

29. Denstel KD, Broyles ST, Larouche R, Sarmiento OL, Barreira TV, Chaput JP, Church TS, Fogelholm M, Hu G, Kuriyan R, et al. Active school transport and weekday physical activity in 9-11-year-old children from 12 countries. Int J Obes Suppl. 2015;5(Suppl 2):S100-6.

30. Faulkner GE, Buliung RN, Flora PK, Fusco C. Active school transport, physical activity levels and body weight of children and youth: a systematic review. Prev Med. 2009:48(1):3-8.

31. Pizarro AN, Ribeiro JC, Marques EA, Mota J, Santos MP. Is walking to schoo associated with improved metabolic health? Int J Behav Nutr Phys Act. 2013;10:12.

32. Faulkner G, Stone M, Buliung R, Wong B, Mitra R. School travel and children's physical activity: a cross-sectional study examining the influence of distance. BMC Public Health. 2013;13:1166.

33. Hallal PC, Andersen LB, Bull FC, Guthold R, Haskell W, Ekelund U. Global physical activity levels: surveillance progress, pitfalls, and prospects. Lancet. 2012;380(9838):247-57

34. Peltzer K, Pengpid S. Leisure time physical inactivity and sedentary behaviour and lifestyle correlates among students aged 13-15 in the Association of Southeast Asian Nations (ASEAN) member states, 2007-2013. Int J Environ Res Public Health. 2016;13(2):217.

35. Santos R, Mota J, Okely AD, Pratt M, Moreira C, Coelho-e-Silva MJ, Vale S, Sardinha LB. The independent associations of sedentary behaviour and physical activity on cardiorespiratory fitness. Br J Sports Med. 2014;48(20): 1508-12.

36. Biddle SJ, Gorely T, Marshall SJ, Cameron N. The prevalence of sedentary behavior and physical activity in leisure time: a study of Scottish adolescents using ecological momentary assessment. Prev Med. 2009;48(2): 151-5.

37. lannotti RJ, Wang J. Patterns of physical activity, sedentary behavior, and diet in U.S. adolescents. J Adolesc Health. 2013;53(2):280-6.

38. Steele RM, van Sluijs EM, Sharp SJ, Landsbaugh JR, Ekelund U, Griffin SJ. An investigation of patterns of children's sedentary and vigorous physical activity throughout the week. Int J Behav Nutr Phys Act. 2010;7:88.

39. Jalali-Farahani S, Amiri P, Chin YS. Are physical activity, sedentary behaviors and sleep duration associated with body mass index-for-age and healthrelated quality of life among high school boys and girls? Health Qual Life Outcomes. 2016:14:30.

40. United States Department of Labor: Bureau of Labor Statistics. Average hours per day spent in selected activities by age. https://www.bls.gov/ charts/american-time-use/activity-by-age.htm.

41. LeBlanc AG, Broyles ST, Chaput JP, Leduc G, Boyer C, Borghese MM, Tremblay MS. Correlates of objectively measured sedentary time and selfreported screen time in Canadian children. Int J Behav Nutr Phys Act. 2015; 12:38.

42. Pate RR, Mitchell JA, Byun W, Dowda M. Sedentary behaviour in youth. Br J Sports Med. 2011;45(11):906-13.

43. Foley LS, Maddison R, Jiang Y, Olds T, Ridley K. It's not just the television: survey analysis of sedentary behaviour in New Zealand young people. Int J Behav Nutr Phys Act. 2011;8:132.

44. Neto FA, Eto FN, Pereira TSS, Carletti L, Molina MCB. Active and sedentary behaviours in children aged 7 to 10 years old: the urban and rural contexts, Brazil. BMC Public Health. 2014;14:1174.

45. Atkin AJ, Corder K, Ekelund U, Wijndaele K, Griffin SJ, van Sluijs EM. Determinants of change in children's sedentary time. PLoS One. 2013;8(6): e67627
46. Asare M, Danquah SA. The relationship between physical activity, sedentary behaviour and mental health in Ghanaian adolescents. Child Adolesc Psychiatry Ment Health. 2015;9:11.

47. Sallis JF, Saelens BE. Assessment of physical activity by self-report: status, limitations, and future directions. Res Q Exerc Sport. 2000; 71(Suppl 2):1-14.

\section{Publisher's Note}

Springer Nature remains neutral with regard to jurisdictional claims in published maps and institutional affiliations.
Ready to submit your research? Choose BMC and benefit from:

- fast, convenient online submission

- thorough peer review by experienced researchers in your field

- rapid publication on acceptance

- support for research data, including large and complex data types

- gold Open Access which fosters wider collaboration and increased citations

- maximum visibility for your research: over $100 \mathrm{M}$ website views per year

At $\mathrm{BMC}$, research is always in progress.

Learn more biomedcentral.com/submissions 